\title{
Selective Ion-Molecule Reactions of Lactams with Dimethyl Ether Ions
}

\author{
Tracy Donovan McCarley and Jennifer Brodbelt \\ Department of Chemistry and Biochemistry, University of Texas, Austin, Texas, USA
}

\begin{abstract}
The ion-molecule reactions of dimethyl ether ions $\mathrm{CH}_{3} \mathrm{OCH}_{2}^{+}$and $\left(\mathrm{CH}_{3} \mathrm{OCH}_{3}\right) \mathrm{H}^{+}$, and four- to seven-membered ring lactams with methyl substituents in various positions were characterized by using a quadrupole ion trap mass spectrometer and a triple-quadrupole mass spectrometer. In both instruments, the lactams were protonated by dimethyl ether ions and formed various combinations of $[M+13]^{+},[M+15]^{+}$, and $[M+45]^{+}$adduct ions, as well as unusual $[M+3]^{+}$and $[M+16]^{+}$adduct ions. An additional $[M+47]^{+}$adduct ion was formed in the conventional chemical ionization source of the triple-quadrupole mass spectrometer. The product ions were isolated and collisionally activated in the quadrupole ion trap to understand formation pathways, structures, and characteristic dissociation pathways. Sequential activation experiments were performed to elucidate fragment ion structures and stepwise dissociation sequences. Protonated lactams dissociate by loss of water, ammonia, or methylamine; ammonia and carbon monoxide; and water and ammonia or methylamine. The $[\mathrm{M}+16]^{+}$products, which are identified as protonated lactone structures, are only formed by those lactams that do not have an $N$-methyl substituent. The ion-molecule reactions of dimethyl ether ions with lactams were compared with those of analogous amides and lactones. (J Am Soc Mass Spectrom 1993, 4, 352-361)
\end{abstract}

A variety of molecules containing a lactam ring system are essential in the polymer and pharmaceutical industries. For example, e-caprolactam is a monomer in the ring-opening polymerization of nylon [1], and many antibiotics, such as the penicillins, cephalosporins, and cytochalasins, are based on a lactam ring [2]. Because of the industrial importance of lactams, their analysis has been the focus of many studies.

For nearly three decades, the mass spectral fragmentation of lactam ions has been studied to differentiate structural isomers and correlate fragmentation patterns with structural features [3-25]. The fragmentation of unsubstituted and substituted three- $[3,4]$, four- $[5,6]$, five- $[7-9]$, six- $[8,9]$, and seven-membered $[9,10]$ ring lactams ionized by electron impact ionization has received the most attention. These lactam molecular ions commonly fragment by ring cleavage, producing ketene-type ions or neutrals and/or isocyanate-type ions or neutrals. Ring cleavage may also result in the loss of $\mathrm{CO}, \mathrm{C}_{2} \mathrm{H}_{4}$, or $\mathrm{CH}_{2} \mathrm{~N}$. Fragmentation patterns vary, depending on ring size and the nature and position of the substituents. The fragmentation patterns of $\beta$-lactam antibiotics [11-17], steroidal lactams [18-20], and other complex lactams [21-25] following electron impact ionization, chemical ioniza-

Address reprint nequests to Jentifer Brodbelt, Department of Chemistry and Biochernistry, University of Texas, Austin, TX 78712-1167. tion, fast-atom bombardment ionization, and collisional activation have also been reported; however, the bimolecular reactions of simple lactams and dissociation reactions of the reaction products have not been studied systematically.

In this study, the selectivity of ion-molecule reactions of four- to seven-membered ring lactams, both unsubstituted and with methyl substituents in various positions (Figure 1), with dimethyl ether ions was characterized to correlate reactivity with ring size and substitution pattem. Dimethyl ether ions, $\mathrm{CH}_{3} \mathrm{OCH}_{2}^{+}$ and $\left(\mathrm{CH}_{3} \mathrm{OCH}_{3}\right) \mathrm{H}^{+}$, which have previously been shown to react selectively with many organic compounds [26, 27], were used for chemical ionization to form protonated lactams and adduct ions in both a quadrupole ion trap mass spectrometer and a triplequadrupole mass spectrometer. The product ions were collisionally activated in the ion trap mass spectrometer to induce dissociation for elucidation of formation pathways, structures, and dissociation pathways, and the identities of the eliminated neutrals were determined by sequential activation experiments. As is described herein, the $N$-methyl lactams show distinctive reaction pathways, compared with their $C$-methyl counterparts.

The ion-molecule reactions of lactams have also been compared with those of their acyclic counterparts (amides) and their ester analogs (lactones). The latter have been characterized by this laboratory in an earlier 
<smiles>O=C1CCN1</smiles>

2-azetidinone<smiles>CN1CCCC1=O</smiles>

1-methyl-2-pyrrolidinone<smiles>CC1CCC(=O)N1</smiles>

5-methyl-2-pyrrolidinone<smiles>O=C1CCCCN1</smiles>

2-piperidone

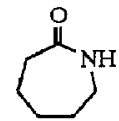

$\varepsilon$-caprolactam

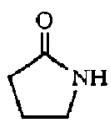

2-pyrrolidinone<smiles>CC1CCNC1=O</smiles>

3-methyl-2-pyrrolidinone<smiles>CC1CCC(=O)N1C</smiles>
1,5-dimethyl-2-pyrolidinone<smiles>CN1CCCCC1=O</smiles>

1-methyl-2-piperidone

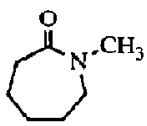

$N$-methyl- $\varepsilon$-caprolactam

Figure 1. Lactam structures.

work [28]. In that study, reactions of dimethyl ether ions with lactones produced protonated lactones as well as adduct ions corresponding to $[\mathrm{M}+15]^{+},[\mathrm{M}+$ $45]^{+}$, and $[\mathrm{M}+47]^{+}$. Protonated lactones dissociate primarily by loss of water sequential loss of water and carbon monoxide, and neutral losses of a ketene, alkene, and $\mathrm{C}_{2} \mathrm{H}_{4} \mathrm{O}$.

\section{Experimental}

Unless otherwise noted, experiments were performed in a Finnigan quadrupole ion trap mass spectrometer (Finnigan-MAT, San Jose, CA) [29]. Samples were introduced into the vacuum chamber through a variable leak valve or a direct-inlet probe to a nominal pressure of $0.8-2.0 \times 10^{-6}$ torr; dimethyl ether was used as a reagent gas at a pressure of $1 \times 10^{5}$ torr; and helium was admitted as a buffer gas at 1 mtorr (corrected gauge pressure measured by an ionization gauge attached to the vacuum chamber). The reactive ions of dimethyl ether are methoxymethylene, $\mathrm{CH}_{3} \mathrm{OCH}_{2}^{+}$, at $m / z \quad 45$, and protonated dimethyl ether, $\left(\mathrm{CH}_{3} \mathrm{OCH}_{3}\right) \mathrm{H}^{+}$, at $\mathrm{m} / z$ 47. After an ion-molecule reaction period of $50-100 \mathrm{~ms}$, the product ion of interest was isolated by apex isolation [30] consisting of a $-100-200-V$ direct-current potential combined with an appropriate radio frequency voltage applied to the ring electrode. Isolated ions were then collisionally activated by a supplementary alternating-current tickle voltage applied across the end caps, typically $0.2-0.4$ $\mathrm{V}_{p-p}$ for $7-12 \mathrm{~ms}$ at a $q_{z}$ value of 0.4 . Dimethyl- $d_{6}$ ether was used as a reagent gas at $1 \times 10^{-5}$ torr for deuterium-labeling experiments.

For experiments performed in a Finnigan TSQ-70 triple-quadrupole mass spectrometer, samples were introduced with a direct-inlet probe. Dimethyl ether was used as a reagent gas at 1.4 torr in a chemical ionization source region that was maintained at $150^{\circ} \mathrm{C}$. The dimethyl ether pressure was measured by a Pirani gauge located in the reagent gas line outside the vacuum manifold. Ions were mass analyzed by the first quadrupole.

All samples were purchased from Aldrich Chemical Co. (Milwaukee, WI) and were used without further purification. Purities were tested by electron impact and isobutane chemical ionization, as well as gas chromatography/mass spectrometry. Dimethyl ether was purchased from MG Industries and dimethyl- $d_{6}$ ether from Isotec, Inc.

\section{Results and Discussion}

\section{Bimolecular Reactions of Lactams with Dimethyl Ether Ions}

The ion-molecule reaction products of the lactams with dimethyl ether reagent ions in the ion trap are summarized in Table 1. All of the lactams were protonated by dimethyl ether reagent ions and formed [ $\mathrm{M}$ $+15]^{+}$adduct ions by methyl cation transfer, and most of the lactams formed $[M+45]^{+}$adduct ions. Other products formed include $[\mathrm{M}+3]^{+},[\mathrm{M}+13]^{+}$, and $[\mathrm{M}+16]^{+}$adducts. To determine which dimethyl ether reagent ion is responsible for formation of each adduct ion, the reagent ions at $\mathrm{m} / z$ 45 (the methoxymethylene cation) and $m / z 47$ (protonated dimethyl ether) were individually isolated and allowed to react with the lactams. Protonated dimethyl ether resulted only in protonation of the lactams, whereas the methoxymethylene cation produced all of the adducts listed in Table 1 as well as a small amount of the protonated molecules.

Although simple protonation or formation of [M + $15]^{+}$adducts shows no dependence on ring size or substituent position, formation of the $[M+13]^{+}$and $[\mathrm{M}+16]^{+}$adducts appears to be highly selective. For instance, of the five-, six- and seven-membered ring systems, the $[M+13]^{+}$and $[M+16]^{+}$adducts were formed only by lactams that do not have a methyl 
Table 1. Ion-molecule reaction products of lactams and dimethyl ether ions

\begin{tabular}{|c|c|c|c|c|c|}
\hline \multirow[b]{2}{*}{ Lactam $(M W)^{\mathrm{a}}$} & \multicolumn{5}{|c|}{ Reaction products (relative abundance) } \\
\hline & {$[\mathrm{M}+\mathrm{H}]^{+}$} & {$[M+13]^{+}$} & {$[M+15]^{+}$} & {$[M+16]^{+}$} & {$[M+45]^{+}$} \\
\hline 2-Azetidinone $(71)^{b}$ & $50 \%$ & $30 \%$ & 5\% & $0 \%$ & $0 \%$ \\
\hline 2-Pyrrolidinone (85) & $70 \%$ & $<5 \%$ & $20 \%$ & $5 \%$ & $<5 \%$ \\
\hline 1-Methyl-2-pyrrolidinone (99) & $\mathbf{5 5 \%}$ & $0 \%$ & $40 \%$ & $0 \%$ & $5 \%$ \\
\hline 3-Methyl-2-pyrrolidinone (99) & $45 \%$ & $10 \%$ & $30 \%$ & $10 \%$ & $5 \%$ \\
\hline 5-Methyl-2-pyrrolidinone (99) & $50 \%$ & $5 \%$ & $30 \%$ & $10 \%$ & $5 \%$ \\
\hline 1,5-Dimethyl-2-pyrrolidinone (113) & $60 \%$ & $0 \%$ & $35 \%$ & $0 \%$ & $5 \%$ \\
\hline 2-Piperidane (99) & $55 \%$ & $<5 \%$ & $35 \%$ & $<5 \%$ & $5 \%$ \\
\hline 1-Methyl-2-piperidone $(113)$ & $20 \%$ & $0 \%$ & $55 \%$ & $0 \%$ & $25 \%$ \\
\hline E-Caprolactam (113) & $60 \%$ & $5 \%$ & $20 \%$ & $5 \%$ & $10 \%$ \\
\hline$N$-Methyl-e-caprolactam (127) & $20 \%$ & $0 \%$ & $25 \%$ & $0 \%$ & $55 \%$ \\
\hline
\end{tabular}

\footnotetext{
${ }^{a} M W$, molecular weight

b The remaining $15 \%$ of the total ion current was contributed by an $\mathrm{IM}+3 \mathrm{I}^{+}$adduct, see text for a discussion of this process.
}

substituent on the nitrogen atom. For lactams with an $N$-methyl substituent, neither $[\mathrm{M}+13]^{+}$nor $[\mathrm{M}+$ $16]^{+}$adducts were formed. Finally, the four-membered ring (2-azetidinone) formed an $[\mathrm{M}+13]^{+}$adduct but not an $[\mathrm{M}+16]^{+}$adduct.

Collisional activation of the $[M+45]^{+}$ions provides information that can help in understanding the selectivity of $[\mathbf{M}+13]^{+}$and $[\mathbf{M}+16]^{+}$formation. In all cases, collisional activation of the $[\mathrm{M}+45]^{+}$ion produced fragment ions that correspond to the [M+ $13]^{+},[M+15]^{+}$, and $[M+16]^{+}$adducts observed in the ion molecule reaction spectrum of each compound (see Table 2). This suggests that the $[\mathrm{M}+45]^{+}$adduct is a precursor in the formation of the other adducts shown in Table 1. Previously, $[\mathrm{M}+45]^{+}$adducts formed from reactions of dimethyl ether ions with organic substrates have been observed as precursors to $[M+13]^{+}$and $[M+15]^{+}$ions through mechanisms in which either methanol or formaldehyde, respectively, is eliminated from the $[M+45]^{+}$precursor ion [26]. The formation of the $[M+13]^{+}$adducts appears to be a type of methylene substitution process known to occur in compounds containing certain heteroatomic sites $\left(-\mathrm{OH},-\mathrm{NH}_{2},-\mathrm{NHR}\right)$ [26, 27, 31]. The reaction proceeds by attack of the heteroatom on the methoxymethylene cation, followed by the spontaneous elimination of $\mathrm{CH}_{3} \mathrm{OH}$. This pathway is only observed when the heteroatom has a hydrogen attached that can be involved in the $\mathrm{CH}_{3} \mathrm{OH}$ loss. Thus, the lactams with $\mathrm{N}$-methyl substituents cannot undergo this type of ion molecule reaction. This selectivity can be observed in Table 1.

Unlike the $[\mathrm{M}+13]^{+}$and $[\mathrm{M}+15]^{+}$adducts, the formation of the $[M+16]^{+}$ion by dissociation of an $[\mathrm{M}+45]^{\mathrm{r}}$ adduct has not been reported prior to this study. To assist in the elucidation of the structure of this intriguing $[M+16]^{+}$adduct, the $[M+16]^{+}$ adduct of each lactam was collisionally activated, and the results are summarized in Table 3 . In the collisionactivated dissociation (CAD) spectra of the $[M+16]^{+}$ ions, all of the fragments appear at odd masses. This indicates that either the $[M+16]^{+}$ions do not contain a nitrogen atom or that all the fragments are radical cations (a rather unlikely possibility for the dissociation of closed-shell ions). For example, the CAD spectrum of the $[M+16]^{+}$adduct of 2-pyrrolidinone shows

Table 2. CAD products of lactam $[M+45]^{+}$adducts

\begin{tabular}{|c|c|c|c|c|c|}
\hline \multirow[b]{2}{*}{ Lactam (MW) } & \multicolumn{5}{|c|}{ Fragment ions (relative abundance) } \\
\hline & {$[\mathbf{M}-15]^{+}$} & $\begin{array}{c}{[\mathrm{M}+2]^{+}} \\
-\mathrm{CH}_{3}-\mathrm{N}=\mathrm{CH}_{2}\end{array}$ & $\begin{array}{l}{[\mathrm{M}+13]^{+}} \\
-\mathrm{CH}_{3} \mathrm{OH} \\
\end{array}$ & $\begin{array}{c}{[\mathrm{M}+15]^{+}} \\
-\mathrm{CH}_{2} \mathrm{O} \\
\end{array}$ & $\begin{array}{r}{[\mathrm{M}+16]^{+}} \\
-\mathrm{NH}=\mathrm{CH}_{2} \\
\end{array}$ \\
\hline 2-Pyrrolidinone (85) & $\mathbf{5} \%$ & $0 \%$ & $25 \%$ & $20 \%$ & $50 \%$ \\
\hline 1-Methyl-2-pyrrolidinone (99) & $0 \%$ & $20 \%$ & $0 \%$ & $80 \%$ & $0 \%$ \\
\hline 3-Methyl-2-pyrrolidinone (99) & $5 \%$ & $0 \%$ & $30 \%$ & $15 \%$ & $50 \%$ \\
\hline 5-Methyl-2-pyrrolidinone (99) & $0 \%$ & $0 \%$ & $10 \%$ & $35 \%$ & $55 \%$ \\
\hline 1,5-Dimethyl-2-pyrrolidinone (113) & $\mathbf{0} \%$ & $5 \%$ & $0 \%$ & $95 \%$ & $0 \%$ \\
\hline 2-Piperidone (99) & $20 \%$ & $0 \%$ & $20 \%$ & $45 \%$ & $15 \%$ \\
\hline 1-Methyl-2-piperidone (113) & $0 \%$ & $5 \%$ & $0 \%$ & $95 \%$ & $0 \%$ \\
\hline E-Caprolactam (113) & $25 \%$ & $0 \%$ & $40 \%$ & $30 \%$ & $5 \%$ \\
\hline N-Methyl-E-caprolactam (127) & $0 \%$ & $5 \%$ & $0 \%$ & $95 \%$ & $0 \%$ \\
\hline
\end{tabular}

${ }^{a} \mathrm{CAD}$, collision-activated dissociation; MW, molecular weight. 
Table 3. CAD products of lactam $[M+16]^{+}$adducts $^{a}$

\begin{tabular}{lllll}
\hline Lactam (MW) & \multicolumn{4}{c}{ Fragment ions (relative abundance) } \\
\hline \hline 2-Pyrrolidinone (85) & $47^{+}(10 \%)^{\mathrm{b}}$ & $55^{+}(15 \%)$ & $59^{+}(60 \%)$ & $83^{+}(15 \%)$ \\
3-Methyl-2-pyrrolidinone (99) & $59^{+}(80 \%)$ & $83^{+}(20 \%)$ & & \\
5-Methyl-2-pyrrolidinone $(99)$ & $55^{+}(55 \%)$ & $73^{+}(15 \%)$ & $83^{+}(30 \%)$ & \\
E-Caprolactam (113) & $69^{+}(25 \%)$ & $97^{+}(75 \%)$ & &
\end{tabular}

${ }^{a}$ CAD, collision-activated dissociation; MW, molecular weight.

'This ion is attributed to protonated dimethyl ether, resulting from an ion-molecule reaction rather than dissociation of the $[M+16]^{+}$adduct

a base peak at $m / z 59$ and less abundant ions at $\mathrm{m} / \mathrm{z}$ 55 and 83 (Figure 2a). This spectrum is strikingly similar to the CAD spectrum of the $[\mathrm{M}+15]^{+}$adduct of $y$-butyrolactone, the lactone analog of 2-pyrrolidinone (Figure 2b). Additional resemblances are found in the CAD spectra of the $[M+16]^{+}$adduct of 3-methyl-2-pyrrolidinone and the $[\mathrm{M}+15]^{+}$adduct of $\alpha$-methyl- $\gamma$-butyrolactone, the $[M+16]^{+}$adduct of 5 methyl-2-pyrrolidinone and the $[\mathrm{M}+15]^{\prime}$ adduct of $\gamma$-valerolactone, and, likewise, the $[\mathrm{M}+16]^{+}$adduct of $\epsilon$-caprolactam and the $[\mathrm{M}+15]^{+}$adduct of $\epsilon$ caprolactone. ${ }^{1}$ On the basis of this observation, the structures of the lactam $[\mathrm{M}+16]^{+}$adducts and the lactone $[\mathrm{M}+15]^{+}$adducts are presumed to be identical.

A mechanism showing the formation of the 5methyl-2-pyrrolidinone $[\mathrm{M}+16]^{+}$adduct is proposed in Scheme Ia. The lactam nitrogen atom attacks the electron-deficient methoxymethylene cation to form an $[\mathrm{M}+45]^{+}$adduct ion, after which a methoxyl group is transferred to the carbonyl carbon in a ring-opening rearrangement. Methoxyl shifts have been observed before in the ion-molecule reactions of methoxymethylene cations with simple olefins and carbonyl compounds [32]. Next, $\mathrm{HN}=\mathrm{CH}_{2}$ is eliminated, followed by an energetically favorable cyclization to yield the final methylated lactone ion. Such a mechanism also accounts for the absence of an $[\mathrm{M}+16]^{+}$adduct in the ion-molecule reaction spectra (and a corresponding fragment ion in the CAD spectra of the $[M+45]^{+}$ adducts) of the lactams that have a methyl-substituted nitrogen atom. If the $[\mathrm{M}+45]^{+}$adducts of these $N$ methyl compounds dissociate according to the mechanism shown in Scheme Ia, then the neutral eliminated would be $\mathrm{CH}_{2}=\mathrm{N}-\mathrm{CH}_{3}$, a loss of $43 \mathrm{u}$, rather than $\mathrm{CH}_{2}=\mathrm{NH}$, and the resulting final product would be observed at $[M+45-43]^{+}$, or $[M+2]^{+}$. In fact, CAD of the $[\mathrm{M}+45]^{+}$adduct of each of the nitrogen-substituted lactams produces an ion at $[M+2]^{+}$(see Table 2), which offers additional support for this unusual process.

The mechanism in Scheme Ia is further substantiated by experiments in which dimethyl- $d_{6}$ ether was substituted for dimethyl ether as the reagent gas. According to this mechanism, 5-methyl-2-pyrrolidinone

\footnotetext{
${ }^{1}$ All CAD spectra of lactone $[M+15]^{+}$ions are summarized in ref 28 , and are thus available for inspection.
}

will react with dimethyl- $d_{6}$ ether ions to produce an $[\mathrm{M}+50]^{+}$adduct at $m / z 149$ rather than an $[\mathrm{M}+45]^{+}$ adduct at $m / z 144$, which would then dissociate by loss of $\mathrm{NH}=\mathrm{CD}_{2}$ to form an $[\mathrm{M}+19]^{+}$ion at $\mathrm{m} / z$ 118. This process is depicted in Scheme $\mathrm{Ib}$, and the complete product spectrum is shown in Figure 3. Reaction of 5-methyl-2-pyrrolidinone with dimethyl- $d_{6}$ ether in the ion trap indeed produced an $[\mathrm{M}+19]^{+}$adduct at $m / z 118$ and an $[\mathrm{M}+50]^{+}$adduct at $m / z 149$, as well as an $[\mathrm{M}+\mathrm{D}]^{+}$adduct at $m / z 101$, an $[\mathrm{M}+15]^{+}$ adduct at $m / z 114$ (this adduct corresponds to the $[M+13]^{r}$ adduct in the dimethyl ether experiments), and an $[\mathrm{M}+18]^{+}$adduct at $m / z 117$ (which corresponds to the $[M+15]^{+}$adduct in the dimethyl ether experiments). The product peaks shown shift exclu-
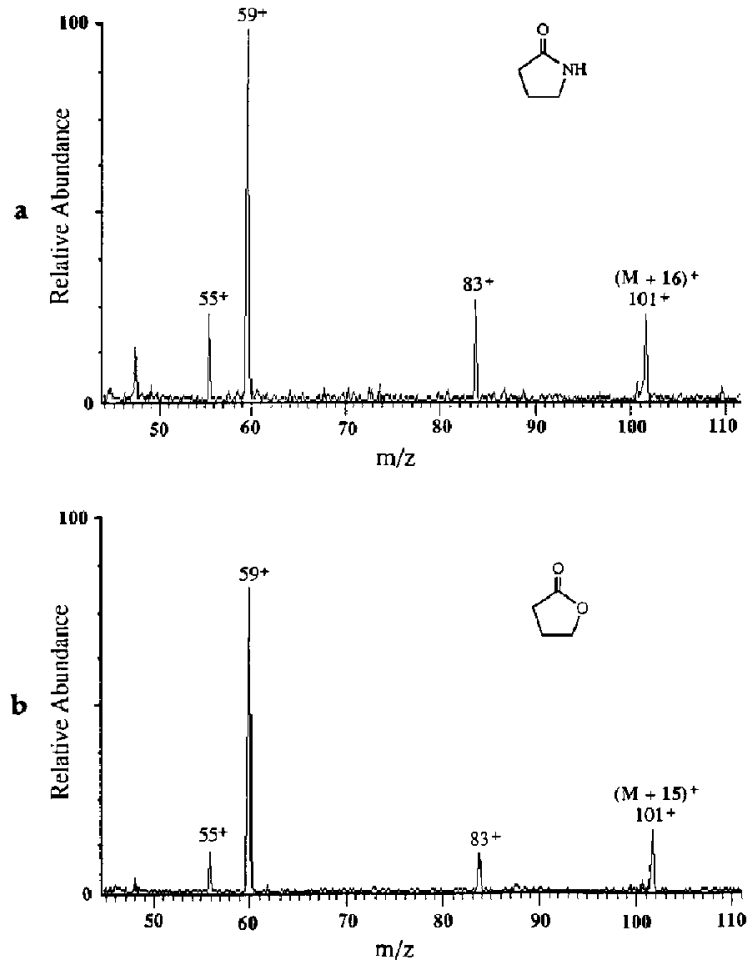

Figure 2. CAD spectra of $(a)$ the $[M+16]^{+}$adduct of 2-pyrrolidinone, and (b) the $[\mathrm{M}+15]^{+}$adduct of $\gamma$-butyrolactone in the ion trap mass spectrometer. 


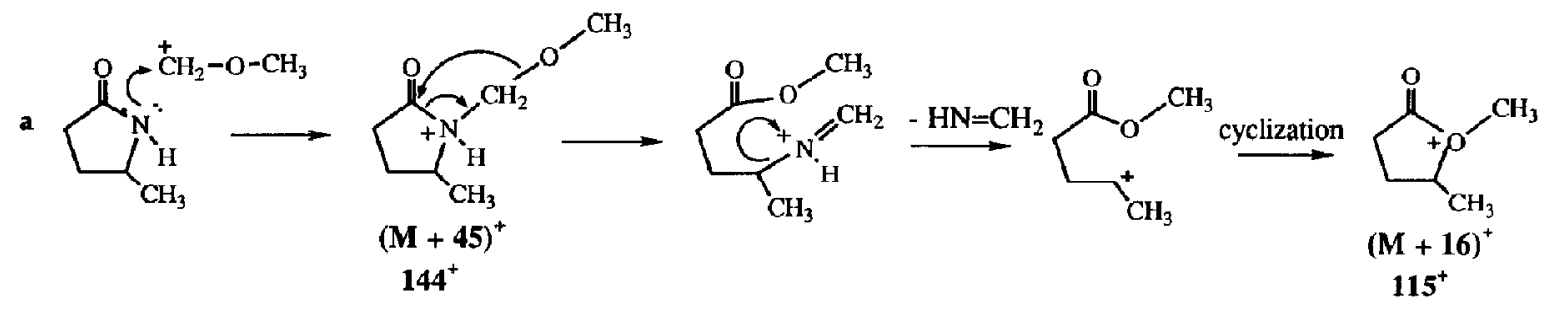

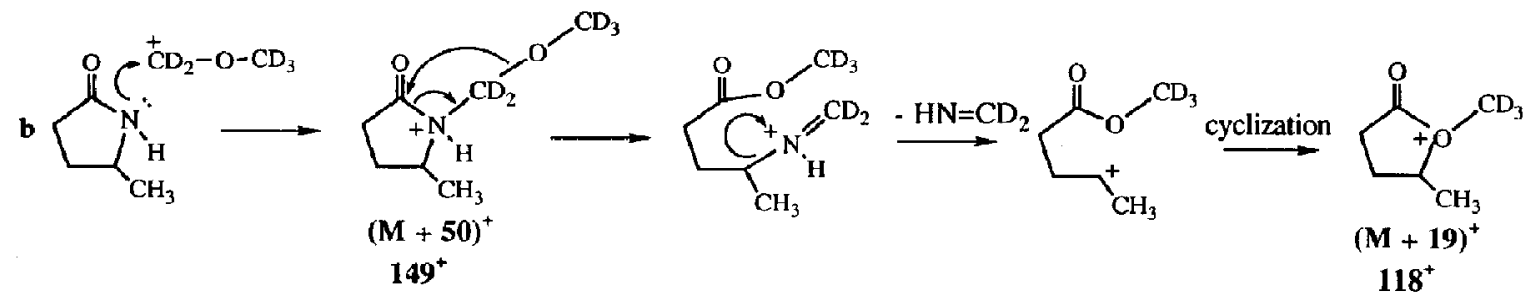

Scheme I. Mechanism for formation of lactam $[M+16]^{+}$adducts by reactions of 5-methyl-2-pyrrolidinone with (a) dimethyl ether ions and (b) dimethyl- $a_{6}$ ether ions.

sively to their fully deuterated counterparts, demonstrating that no atom scrambling occurs.

The four-membered ring lactam, 2-azetidinone, was the only lactam that did not form a stable $[\mathrm{M}+45]^{+}$ adduct, yet it formed an unusual $[\mathrm{M}+3]^{+}$adduct at $m / z 74$. This ion is a reaction product of 2 -azetidinone and the methoxymethylene cation of dimethyl ether. One possible mechanism (shown in Scheme II) for the formation of this ion involves an intermediate [M + $45]^{+}$species that dissociates by elimination of a ketene neutral $\left(\mathrm{CH}_{2}=\mathrm{C}=\mathrm{O}\right)$. The high degree of ring strain of 2-azetidinone may promote this process.

In addition, 2-azetidinone is the only $[\mathrm{M}+13]^{+}$ ion-forming lactam that does not also form the [M+ $16]^{+}$product. The other lactams that formed $[M+13]^{+}$ adducts also formed $[\mathrm{M}+16]^{+}$adducts. This anomaly may be due to the fact that 2-azetidinone has another competing reaction pathway, the formation of $[\mathrm{M}+$

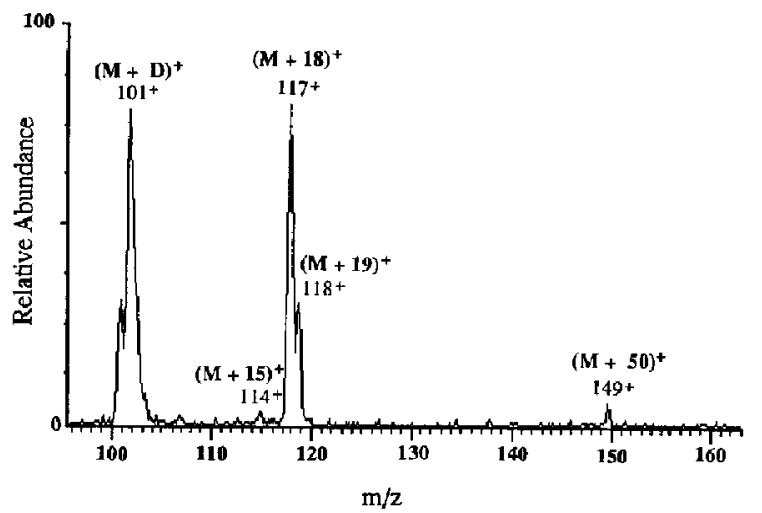

Figure 3. Reaction spectrum of dimethyl- $d_{6}$ ether ions and 5-methyl-2-pyrrolidinone. $3]^{+}$described above, which is kinetically or thermodynamically favored over the formation of $[M+16]^{+}$ ions.

Another interesting feature of the reactivity of 2azetidinone is that at sample pressures greater than $1 \times 10^{-6}$ torr, the products formed by ion-molecule reactions with dimethyl ether ions are obscured by self-chemical ionization reaction products. When 2azetidinone is ionized by electron impact ionization at these higher sample pressures, and in the absence of dimethyl ether, ions are formed at $m / z 72,101,113$, 126 , and 143 . The ion at $m / z 72$ is simply a protonated lactam formed by self-chemical ionization, and the ion at $m / z 143$ is a proton-bound dimer, $\left[2 \mathrm{M}+\mathrm{H}^{+}\right.$. The proton-bound dimer was isolated and activated to determine whether it is a precursor in the formation of any of the other ions, but it showed only one dissociation product that corresponds to protonated 2-azetidinone. The ions at $m / z 101,113$, and 126 are presumably formed by reaction of neutral 2-azetidinone with ions produced by spontaneous elimination of 42,30 , or $17 \mathfrak{u}$, respectively, from protonated lactams. Similar reactions were observed for some of the larger ring lactams at high sample pressures but to a lesser extent than 2-azetidinone. Again, this unique behavior may be caused by a higher degree of ring strain in the four-membered ring than in the larger ring systems, making 2-azetidinone extremely reactive.

\section{Comparison of Reactions in an Ion Trap and a Triple-Quadrupole Mass Spectrometer}

The lactams were also studied in a triple-quadrupole mass spectrometer to observe bimolecular reactions at the higher dimethyl ether pressure of the conventional 


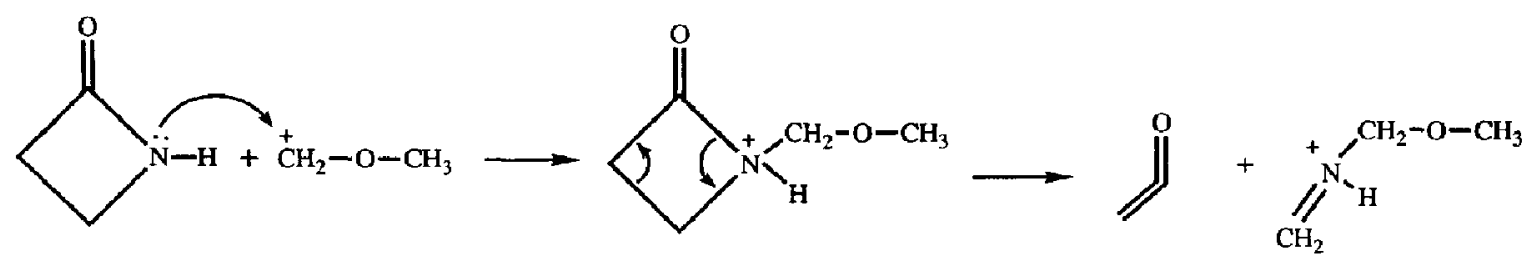

$(\mathbf{M}+45)^{+}$

$(\mathrm{M}+3)^{+}$

$74^{+}$

Scheme II. Proposed mechanism for formation of the 2-azetidinone $[\mathrm{M}+3]^{+}$adduct.

chemical ionization source that is not available in an ion trap. The lactams studied were 2-azetidinone, 2pyrrolidinone, 3-methyl-2-pyrrolidinone, and 5-methyl-2-pyrrolidinone. In each case the same selection of adducts that were formed in the ion trap were also formed in the triple-quadrupole mass spectrometer. In addition, each lactam formed an $[M+47]^{+}$adduct that was not observed in the ion trap experiments. On collisional activation, these $[\mathrm{M}+47]^{+}$adducts dissociated by loss of a neutral dimethyl ether molecule, indicating that they are proton-bound complexes between the lactam and dimethyl ether. This loose complex may be observed in the conventional chemical ionization source and not in the ion trap because dimethyl ether at 1 tor should be more effective at providing third-body collisional stabilization of the loosely bound complex than helium at $1 \mathrm{mtorr}$ in the ion trap. The $[M+45]^{\prime}$ and $[M+47]^{+}$adducts were formed in higher abundance than the other adducts, and the $[M+13]^{+}$and $[M+16]^{+}$adducts of the 2-pyrrolidinones were much lower in intensity relative to the $[M+15]^{+}$ion than they were in the ion trap. The slow time scale of experiments in the ion trap (i.e., $100 \mathrm{~ms}$ compared with $<1 \mathrm{~ms}$ in the conventional ion source) may favor the formation of the $[\mathrm{M}+13]^{+}$and $[M+16]^{+}$products, and likewise the ions formed in the ion trap retain their excess internal energy for a longer time because they are not as rapidly collisionally stabilized.

\section{Comparison of Bimolecular Reactions of Lactams and Amides with Dimethyl Ether Ions}

To evaluate the influence of ring strain on the reactivity of the lactams, the bimolecular reactions of lactams and dimethyl ether ions were compared with those of their acyclic counterparts, the amides. Both $N$-methylacetamide and $N$-ethylacetamide were allowed to react with dimethyl ether ions in the ion trap mass spectrometer under the same conditions as the lactam experiments. Both amides protonated and also formed $[\mathrm{M}+15]^{+}$adducts, but neither formed the $[\mathrm{M}+13]^{+}$, $[M+16]^{+}$, or $[M+45]^{+}$adducts observed for the lactams. On the basis of the mechanism proposed for formation of $[M+13]^{+}$products for other nitrogen- containing organic substrates, the absence of amide $[\mathrm{M}+13]^{+}$ions is somewhat surprising [31]. Each of the amides has an accessible nucleophilic nitrogen site with an available hydrogen atom for the required elimination of $\mathrm{CH}_{3} \mathrm{OH}$. Apparently, the simple methyl cation transfer process is more energetically or kinetically favorable, making the methylene substitution process noncompetitive in the ion trap. The fact that $[\mathrm{M}+16]^{+}$ions are not observed is consistent with the mechanism shown in Scheme Ia, which requires an initially cyclic structure to accommodate the multistep process. The analogous mechanism for acyclic amides would result in splitting of the structure into two parts after the methoxyl transfer, producing a neutral ester moiety and an imine ion. Finally, the absence of [M + $45]^{+}$ions suggests that these ions are not as stable as the corresponding lactam ions and that they spontaneously dissociate to $[M+15]^{+}$ions.

\section{Comparison of Reactions of Lactams and Lactones with Dimethyl Ether Ions}

The reactions of lactams and lactones were compared to evaluate the influence of a nitrogen- versus oxygenring heteroatom. The lactams formed a greater variety of reaction products with dimethyl ether ions than the lactones. The lactones protonated and formed $[\mathbf{M}+$ $15]^{+},[\mathrm{M}+45]^{+}$, and $[\mathrm{M}+47]^{+}$adducts [28], whereas the lactams additionally formed $[\mathrm{M}+3]^{+},[\mathrm{M}+13]^{+}$, and $[M+16]^{+}$adducts, depending on ring size and substituent position. The $[\mathbf{M}+13]^{+}$and $[M+16]^{+}$ adducts presumably are formed through different [M $+45]^{+}$intermediates than the $[M+15]^{+}$adduct. That is, the $[\mathrm{M}+13]^{+}$and $[\mathrm{M}+16]^{+}$ions are produced from $[M+45]^{\dagger}$ precursors in which the methylene group of the $\mathrm{CH}_{3} \mathrm{OCH}_{2}^{+}$ion is covalently attached to the nitrogen heteroatom of the lactam, but the $[M+$ $15]^{+}$ions are formed by $[\mathrm{M}+45]^{+}$precursors in which the methyl group of the $\mathrm{CH}_{3} \mathrm{OCH}_{2}^{+}$ion is directed at a heteroatom.

Another difference in the reactions of lactams and lactones with dimethyl ether ions is the formation of $[\mathrm{M}+45]^{+}$and $[\mathrm{M}+47]^{+}$adducts in the ion trap. Lactams formed $[M+45]^{+}$but not $[M+47]^{+}$adducts in the ion trap, whereas the lactones formed $[\mathrm{M}+47]^{+}$ 


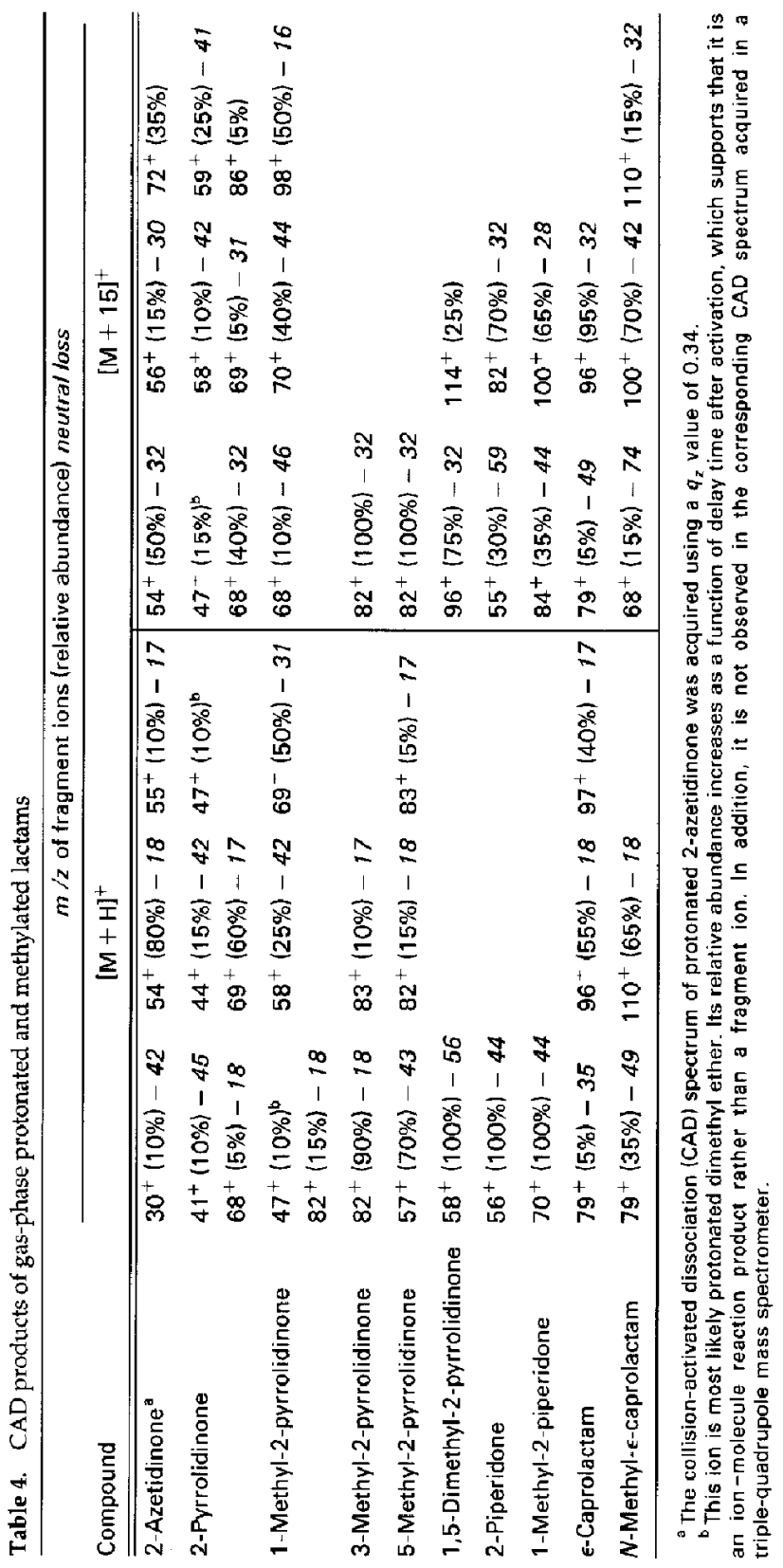


but not $[M+45]^{+}$adducts. In contrast, lactams and lactones formed both types of adducts in the conventional chemical ionization source of the triplequadrupole mass spectrometer. On the basis of the fact that only stable adducts survive in the ion trap environment, apparently the lactam $[\mathrm{M}+45]^{+}$adducts are more stable than the $[\mathrm{M}+47]^{+}$adducts, and the lactone $[\mathrm{M}+47]^{+}$adducts are more stable than the $[\mathrm{M}+$ $45]^{+}$adducts.

\section{Dissociation Reactions of Lactams}

Because the ion-molecule reactions of all the lactams produced $[\mathrm{M}+\mathrm{H}]^{+}$and $[\mathrm{M}+15]^{+}$adduct ions, the dissociation pathways of these two products were characterized by CAD. The results are summarized in Table 4. Some of the neutrals eliminated on activation of protonated lactams include ammonia $\left(\mathrm{NH}_{3}\right)$, water $\left(\mathrm{H}_{2} \mathrm{O}\right)$, methylamine $\left(\mathrm{CH}_{3} \mathrm{NH}_{2}\right), 35,42,44,45,49$, and $56 \mathrm{u}$. The elucidation of the identities of some of these neutrals is described in the following sections.

The loss of $\mathrm{H}_{2} \mathrm{O}$ is the most common fragmentation pathway of protonated lactams. Most of the protonated lactams that dissociate by elimination of $\mathrm{H}_{2} \mathrm{O}$ also dissociate by loss of $\mathrm{NH}_{3}$, if the lactam nitrogen is unsubstituted, or $\mathrm{CH}_{3} \mathrm{NH}_{2}$, if the lactam nitrogen has a methyl substituent, and, typically, the loss of $\mathrm{H}_{2} \mathrm{O}$ is dominant over $\mathrm{NH}_{3}$ or $\mathrm{CH}_{3} \mathrm{NH}_{2}$ loss. The exceptions are protonated 2-pyrrolidinone and 1-methyl-2-pyrrolidinone, in which the losses of $\mathrm{NH}_{3}$ and $\mathrm{CH}_{3} \mathrm{NH}_{2}$, respectively, are the base peaks in the CAD spectra. In the $\mathrm{CAD}$ spectrum of protonated 5-methyl-2-pyrrolidinone, losses of both $\mathrm{H}_{2} \mathrm{O}$ and $\mathrm{CH}_{3} \mathrm{NH}_{2}$ are minor. The only lactams that do not dissociate by loss of $\mathrm{H}_{2} \mathrm{O}$ or $\mathrm{NH}_{3} / \mathrm{CH}_{3} \mathrm{NH}_{2}$ are 1,5-dimethyl-2-pyrrolidinone and the two six-membered rings 2-piperidone and 1-methyl-2 piperidone.

The competition between $\mathrm{H}_{2} \mathrm{O}$ loss and $\mathrm{NH}_{3}$ / $\mathrm{CH}_{3} \mathrm{NH}_{2}$ loss may reflect a thermodynamic or kinetic competition between the oxygen atom and the nitrogen atom as the site of protonation. A recent study reports that $\beta$-lactams are oxygen bases rather than nitrogen bases, but that the gap between the intrinsic basicities of the nitrogen and oxygen is much smaller in $\beta$-lactams than their acyclic counterparts the amides [33]. Therefore, protonation should be thermodynamically favored at the oxygen site but may occur at the nitrogen site also. Presumably, if oxygen-site protonation leads to dehydration, whereas nitrogen-site protonation leads to deamination, dehydration should be favored over deamination. This is consistent with our experimental results, showing that $\mathrm{H}_{2} \mathrm{O}$ loss accounts for $80 \%$ of the total fragment ion current in protonated 2-azetidinone, whereas loss of $\mathrm{NH}_{3}$ accounts for only $10 \%$.

The loss of $45 \mathrm{u}$ from protonated 2-pyrrolidinone can be attributed to a net loss of $\mathrm{CH}_{3} \mathrm{NO}$ by a consecutive or concerted elimination of $\mathrm{NH}_{3}$ and $\mathrm{CO}$. A sequential activation experiment was performed to con-
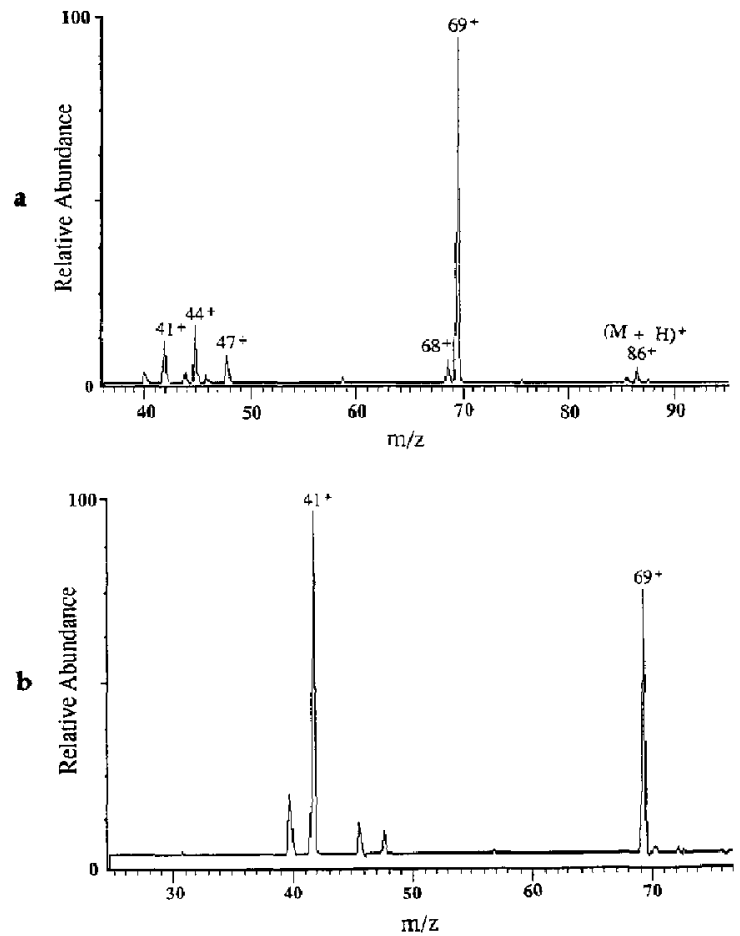

Figure 4. Sequential activation of (a) $m / z$ 86, then (b) $m / z 69$ from the CAD spectrum of protonated 2-pyrrolidinone in the ion trap mass spectrometer.

firm this stepwise loss. After chemical ionization by dimethyl ether ions, protonated 2-pyrrolidinone at $m / z$ 86 was isolated and activated (Figure $4 a$ ). The fragment at $m / z 69$ (loss of $\mathrm{NH}_{3}$ ) was then isolated and activated (Figure $4 b$ ). This yielded an ion at $m / z 41$ by loss of $28 \mathrm{u}$, which confirms a sequential loss of $\mathrm{NH}_{3}$, then $\mathrm{CO}$. Alternatively, this reaction may occur by $\mathrm{CO}$ loss first, but this sequence could not be tested because the CAD spectrum of protonated 2-pyrrolidinone does not contain any ions resulting from $\mathrm{CO}$ luss alone. A mechanism for the sequential loss of $\mathrm{NH}_{3}$ and $\mathrm{CO}$ that is similar to one proposed for $\mathrm{H}_{2} \mathrm{O} / \mathrm{CO}$ loss from protonated lactones [28] is shown in Scheme III. The mechanism is used to illustrate one possible rationalization for the fragmentation, but it has not been confirmed by complete isotopic labeling.

Loss of $\mathrm{NH}_{3}$ and $\mathrm{CO}$ is not observed in the CAD spectra of the other protonated lactams, although this loss from protonated 5-methyl lactams would result in more thermodynamically stabilized product ions. An explanation for this observation is that in the other protonated lactams, competing fragmentation pathways are thermodynamically or kinetically favored over the loss of $\mathrm{NH}_{3}$ and $\mathrm{CO}$. In fact, as a more general observation, several of the fragmentation pathways that are favored by one type of lactam are disfavored or absent for the substituted analogs. It is possible that many of the different dissociation pathways 


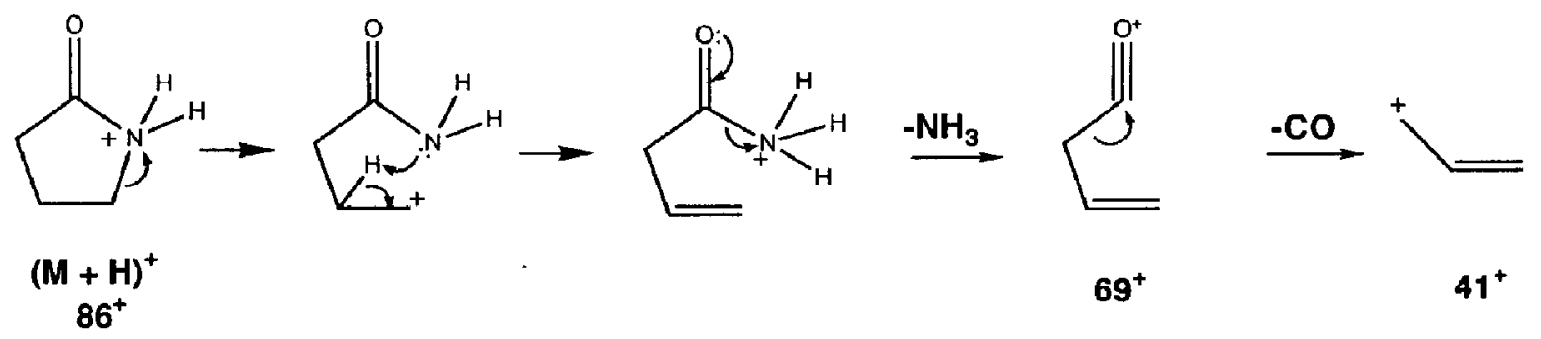

Scheme III. Mechanism for the sequential loss of $\mathrm{NH}_{3}$ and $\mathrm{CO}$ from protonated 2-pyrrolidinone.

have similar activation barriers but have very different kinetic trends; however, the diversity of fragmentation pathways of the protonated lactams is analytically useful because the CAD spectra are especially distinctive.

Sequential activation experiments were used again to elucidate the loss of 49 u from protonated $N$ methyl- $\epsilon$-caprolactam. This net loss of $\mathrm{CH}_{7} \mathrm{NO}$ could reflect a sequential loss of $\mathrm{NH}_{3}$ and $\mathrm{CH}_{3} \mathrm{OH}$ or $\mathrm{CH}_{3} \mathrm{NH}_{2}$ and $\mathrm{H}_{2} \mathrm{O}$. The latter combination seemed more likely because loss of $\mathrm{NH}_{3}$ for the first combination would require extensive rearrangements, including transfer of a methyl group as well as hydrogen atoms. To test the loss of $\mathrm{CH}_{3} \mathrm{NH}_{2}$ and $\mathrm{H}_{2} \mathrm{O}$, the protonated lactam $(m / z 128)$ was isolated and activated to produce ions at $m / z 79$ and 110 (as shown in Table 4). The ion at $m / z 110$, formed by dehydration, was then isolated and activated, which produced an ion at $m / z$ 79. This experiment indicates that sequential loss of $\mathrm{H}_{2} \mathrm{O}$ followed by $\mathrm{CH}_{3} \mathrm{NH}_{2}$ may be induced in the ion trap. Loss of $\mathrm{CH}_{3} \mathrm{NH}_{2}$ followed by loss of $\mathrm{H}_{2} \mathrm{O}$ may also occur, but confirmatory experiments could not be performed because a fragment ion corresponding to loss of $\mathrm{CH}_{3} \mathrm{NH}_{2}$ was not available for sequential activation experiments. Protonated e-caprolactam eliminated $35 \mathbf{u}$ on collisional activation, which may be a similar stepwise loss in which the loss of $\mathrm{CH}_{3} \mathrm{NH}_{2}$ is instead replaced by loss of $\mathrm{NH}_{3}$ because the nitrogen atom does not have a methyl substituent.

The CAD spectra of the lactam $[\mathrm{M}+15]^{+}$adducts do not show as wide a variety of fragmentation pathways as the protonated lactams. The most prominent dissociation is loss of methanol, which is analogous to loss of $\mathrm{H}_{2} \mathrm{O}$ from the simple protonated lactams. The $[\mathrm{M}+15]^{+}$adducts of 1-methyl-2-pyrrolidinone and 1-methyl-2-piperidone are the only ones that do not dissociate by this pathway. The expected loss of $\mathrm{CH}_{3} \mathrm{NH}_{2}$, which is analogous to the common loss of $\mathrm{NH}_{3}$ from protonated lactams, is only observed in the CAD spectrum of the $[\mathrm{M}+15]^{+}$adduct of 2-pyrrolidinone, one of the lactams that exhibits predominant loss of $\mathrm{NH}_{3}$ from the protonated species. One explanation for the absence of $\mathrm{CH}_{3} \mathrm{NH}_{2}$ losses is that the oxygen atom is thermodynamically a more favorable site for methyl cation attachment than the nitrogen atom. $^{2}$ The subsequent dissociation would be limited to loss of methanol because a loss of $\mathrm{CH}_{3} \mathrm{NH}_{2}$ or dimethylamine would require skeletal rearrangement. An alternative explanation is that methyl cation transfer occurs initially at both the oxygen and nitrogen sites, but the intramolecular proton abstraction required for the loss of $\mathrm{CH}_{3} \mathrm{NH}_{2}$ is unfavorable for the nitrogen methylated cation.

As mentioned in an earlier discussion, the [M+ $16]^{+}$ions dissociate by pathways that are analogous to methylated lactone ions [28]; thus, these are not further described.

\section{Conclusions}

The ion-molecule reactions of lactams with dimethyl ether ions show a high degree of selectivity that was not observed in the ion-molecule reactions of lactones or amides with dimethyl ether ions. All of the lactams were protonated by dimethyl ether ions, but the formation of additional adduct ions shows selectivity on the basis of ring size and the presence or absence of a methyl substituent on the lactam nitrogen atom. The $[\mathrm{M}+45]^{+}$adduct ions are the precursors to $[\mathrm{M}+13]^{+}$, $[M+15]^{+}$, and $[M+16]^{+}$adducts that are formed through different intermediate structures and competitive dissociation channels.

The protonated lactams dissociate to yield fragments that are structurally informative. Some of the protonated lactams dissociate by loss of ammonia and sequential loss of ammonia and carbon monoxide, which are analogous to dissociation pathways that are common in protonated lactones. Other fragmentation pathways of protonaled laclams include loss of ammo$\mathrm{nia} /$ methylamine and sequential loss of water and ammonia/methylamine. Each protonated lactam exhibils a unique set of dissociation pathways and product ions, making the discrimination of structural isomers possible.

\footnotetext{
${ }^{2}$ Heats of formation of oxygen and nitrogen protonated and methylated lactams were calculated by generating and minimizing structures with PC MODEL (version 4.4) and then entering the coordinates into the semiempirical program MOPAC (version 6.0). The AM1 hamiltonian operator and default parameters were used in all cases. and a molecular mechanics correction was not used. These preliminary calculations showed that the oxygen atom is the more thermodynamically favorable site for both protonation and methylation.
} 


\section{Acknowledgments}

This work was supported by grants from the Welch Foundation (F-1155), ACS-PRF (25485AC5), NSF (CHE-9122699), and American Cyanamid.

\section{References}

1. Allonck, H. R.; I ampe, F. W. Contemporany Polymer Chemistry: Prentice-Hall: Englewood Cliffs, NJ, 1981; pp 128-130.

2. Betina, V. The Chemistry and Biology of Antibiotics; Elsevier: Amsterdam, 1983; Chapter 4.

3. Baumgarten, H. E.; Parker, R. G.; von Minden, D. L. Org. Mass Spectrom. 1969, 2, 1221.

4. Lengyel, I; Uliss, D. B.; Nafissi-V., M. M.; Sheehan, J. C. Org. Mass Spectron. 1969, 2, 1239.

5. Manhas, M. S.; Ghosh-Mazumdar, B. N.; Bose, A. K. Chem. Commun. 1967, 7, 349.

6. Jackson, M. B: Spotswood. T. M.: Bowie, J. H. Org. Mass Spectrom. 1968, 1, 857.

7. Duffield, A. M.; Budzikiewicz, H.; Djerassi, C. I. Am. Chem. Soc. 1965, 87, 2913.

8. Duffield, A. M.; Budzikiewicz, H.; Djerassi, C. J. Am. Chem. Soc. $1964,86,5536$.

9. Smith, M. B.; Zezza, C. A. Org. Mass Spectrom. 1988, 23, 285.

10. Mitera, J.; Kubelka, V. Org. Mass Spectrom. 1971, 5, 651.

11. Mitscher, H. D.; Showalter, H.; Shirahata, K.; Foltz, R. L. I. Antibiot. (Tokyo) 1975, 28, 668.

12. Müller, M. D.; Seibl, J.; Simon, W. Anal. Chim. Acta 1978, $100,263$.

13. Gower, J. L.; Beaugrand, C.; Sallot, C. Biomed. Environ. Mass Spectrom. 1981, 8, 36.

14. Barber, M.; Bordoli, R. S.; Sedgwick, R. D.; Tyler, A. N.; Green, B. N.; Parr, V. C.; Gower, J. L. Biomed. Environ. Mass Spectrom. 1982, 9, 11.

15. Barbalas, M. P; McLafferty, F. W.; Occolowitz, J. L. Biomed. Environ. Mass Spectrom. 1983, 10, 258.
16. Gower, J. L. Int. J. Mass Spectrom. Ion Phys. 1983, 46, 431.

17. Das, P. R.; Pramanik, B. N.; Girijavallabhan, V. M.; Ganguly, A. K. Org. Mass Spectrom. 1991, 26, 703.

18. Budzikiewicz, H.; Djerassi, C.; Williams, D. H. Mass Spectrometry of Organic Compounds; Holden-Day: San Francisco, 1967; pp. 356-357.

19. Budzikiewicz, V. H.; Compernolle, F.; Van Cauwenberghe, K.; Schulze, K,; Wolf, H.; Quinkert, G. Tefrahedron 1968, 24 , 6797.

20. Ahmad, G.; Mushgiq, M. Org. Mass Spectrom. 1972, 6, 1109.

21. Bujtas, G.; Tamas, J.; Kajtar, M.; Hollosi, M.; Majer, Z. Org. Mass Spectrom. 1982, 17, 398.

22. Majchrzak-Kuczynska, U.; Wiewiorowski, M.; Wyrzykiewicz, E. Org. Mass Spectrom. 1983, 19,600.

23. Pihlaja, K.; Vainiotalo, P.; Bernath, G.; Condos, G.; Gera, L. Org. Mass Spectrom. 1986, 21, 55.

24. St. Georgiev, V.; Coomber, D. C.; Mullen, G. B. Org. Mass Spectrom. 1988, 23, 283.

25. de Petris, G. Org. Mass Spectrom. 1989, 24, 514.

26. Brodbelt, J; Liou, J.; Donovan, T. Anal. Chem. 1991, 63, 1205.

27. Eichmann, E.; Alvarez, E.; Brodbelt, J. 1. Am. Soc. Mass Spectrom. 1992, 3, 5.35.

28. Donovath, T.; Brodbelt, J. I. Am. Soc. Mass Spectrom. 1992, 3, 47.

29. Louris, J. N.; Cooks, R. G.; Syka, J. E. P.; Kelley, P. E.; Stafford, G. C., Jr.; Todd, J. F. J. Anal. Chem. 1987, 59, 1677.

30. Weber-Grabau, M.; Kelley, P. E.; Syka, J. E. P.; Bradshaw, S. C.; Brodbelt, J. S. Proceedings of the 35th ASMS Conference on Mass Spectrometry and Allied Topics; Denver, $\mathrm{CO}, 1987, \mathrm{p}$ 114.

31. Donovan, T.; Brodbelt, J. Biol. Mass Spectrom. 1992, 21, 254.

32. van Doorn, R.; Nibbering, N. M. M. Org. Mass Spectront. 1978, 13, 527.

33. Abbout, J.-L. M.; Canada, T.; Homan, H.; Notario, R.; Cativiela, C.; Diaz de Villegas, M. D.; Bordeje, M. C.; Mo, O; Yanez, M. J. Am. Chem. Soc. 1992, 114, 4728. 